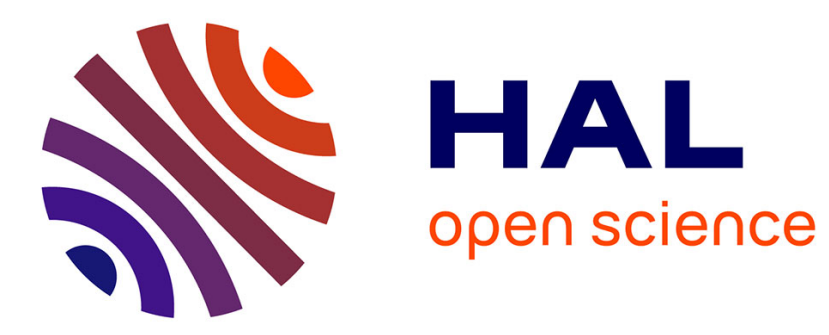

\title{
Iterative Mercury/waterfilling for Parallel Multiple Access Channels
}

He Gaoning, Sophie Gault, Merouane Debbah, Eitan Altman

\section{To cite this version:}

He Gaoning, Sophie Gault, Merouane Debbah, Eitan Altman. Iterative Mercury/waterfilling for Parallel Multiple Access Channels. ICC 2008, May 2008, China. 5 p. hal-00328178

\section{HAL Id: hal-00328178}

https://hal-centralesupelec.archives-ouvertes.fr/hal-00328178

Submitted on 9 Oct 2008

HAL is a multi-disciplinary open access archive for the deposit and dissemination of scientific research documents, whether they are published or not. The documents may come from teaching and research institutions in France or abroad, or from public or private research centers.
L'archive ouverte pluridisciplinaire HAL, est destinée au dépôt et à la diffusion de documents scientifiques de niveau recherche, publiés ou non, émanant des établissements d'enseignement et de recherche français ou étrangers, des laboratoires publics ou privés. 


\section{Iterative Mercury/waterfilling for Parallel Multiple Access Channels}

\author{
Gaoning He, Sophie Gault \\ Motorola Labs \\ 91193 Gif-sur-Yvette - FRANCE \\ Email: gaoninghe@ motorola.com \\ sophie.gault@motorola.com
}

\author{
Mérouane Debbah \\ Supélec \\ 91193 Gif-sur-Yvette - FRANCE \\ Email: merouane.debbah@supelec.fr
}

\author{
Eitan Altman \\ INRIA \\ 06902 Sophia Antipolis - FRANCE \\ Email: eitan.altman@sophia.inria.fr
}

\begin{abstract}
This paper describes a power allocation strategy for fixed constellation over parallel Gaussian channels in the multiuser context. The criterion under consideration is mutual information, given arbitrary input distributions over users and over subcarriers. The algorithm achieves with very low complexity the multi-user aggregate sum mutual information upper bound. The algorithm is based on an iterative Mercury/waterfilling procedure. Moreover, we extend the framework to a decentralized scenario using a linear approximation of the MMSE function. We show, in particular that each user can, under certain assumptions, independently determine the power allocation without knowing the channel information of other users. Simulation results validate the theoretical claims.
\end{abstract}

\section{INTRODUCTION}

Orthogonal Frequency Division Multiplexing (OFDM) is now considered in many standards for transmitting data at high spectral efficiency. This technique is instrumental in the physical layer of several well-known high speed data transmission systems such as wireless local area network (WLAN) or Digital Subscriber Line (xDSL). In order to increase the spectral efficiency, power allocation can be used as an additional degree of freedom [1]-[3]. For a given limited power budget, when the capacity is considered as the optimization criterion, the optimal solution is given by the well-known waterfilling algorithm [4]. However, since capacity is achieved in the context of Gaussian channels with Gaussian inputs, reaching capacity implies the use of Gaussian constellations while these can never be used in practice. Therefore, waterfilling is generally followed by a bit-loading step which consists in deriving for each subcarrier the constellation to use, subject to a target bit error rate. In its actual form, waterfilling is inherently suboptimal. For this reason, mutual information should be a more relevant criterion than capacity. The problem of optimizing power allocation in order to maximize mutual information has been solved in [5] using the Mercury/waterfilling principle. In this paper, we extend this power allocation policy to the multiuser context. Our algorithm presents some similarity with multiuser waterfilling, as implemented in [6].

The paper is organized in the following form: the problem formulation is introduced in section II. In section III, we derive the optimal solution and prove the convergence and

This work is supported by the French grant ANR/WiNEM

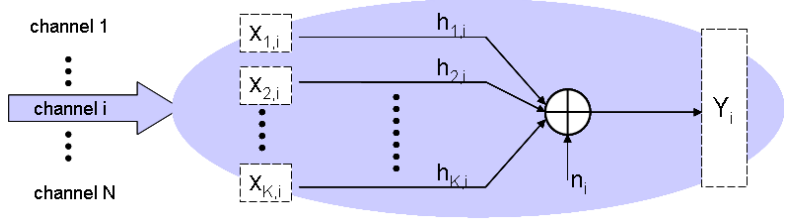

Fig. 1. $\quad N$ parallel Gaussian multiple access channels.

the optimality of the proposed algorithm. In section IV, we provide a decentralized implementation of the power allocation policy. Finally, numerical results are provided in section V followed by conclusions in section VI.

\section{Problem fomulation}

\section{A. OFDM model}

We consider $N$ parallel Gaussian multiple access channels (MAC) with $K$ users in the system as depicted in Fig. 1. These parallel channels can be treated as different subchannels in a multi-carrier system, or the eigenmodes of a MIMO system in the uplink transmission. At the transmitter side, the signal $x_{k, i}$ transmitted on the $i^{t h}$ subcarrier by user $k$ to the base station (BS) is the normalized (i.e. unit-power) symbol $s_{k, i}$ balanced by some power coefficient $p_{k, i}: x_{k, i}=\sqrt{p_{k, i}} s_{k, i}$, where the symbols $\left\{s_{k, i}\right\}$ are usually drawn from some discrete constellations (i.e. PSK, QAM). At the receiver side, $y_{i}$ represents the received signal on the $i^{t h}$ channel, which is the sum of the contributions received from all users

$$
y_{i}=\sum_{k=1}^{K} h_{k, i} x_{k, i}+n_{i}=\sum_{k=1}^{K} h_{k, i} \sqrt{p_{k, i}} s_{k, i}+n_{i}
$$

where $h_{k, i}$ represents the complex gain of the $i^{\text {th }}$ subchannel between user $k$ and the $\mathrm{BS}$, and the noise $n_{i}$ is a zeromean unit-variance complex Gaussian random variable which is independent of the noise on the other channels. Since MAC correspond to the uplink case, individual power constraints have to be taken into account, which can be expressed as ${ }^{1}$

$$
\sum_{i=1}^{N} p_{k, i} \leq P \quad \forall k .
$$

\footnotetext{
${ }^{1}$ To alleviate notations and w.l.o.g., all users are here assumed to be subject to the same power constraint $P$.
} 


\section{B. Power allocation policy}

We tackle the problem of finding the optimal power allocation maximizing the aggregate sum rate that can be reached under the assumption of arbitrary input constellations, while satisfying (1). Since this aggregate sum rate [7], [8] satisfies

$$
\sum_{i=1}^{N} \sum_{k=1}^{K} R_{k, i} \leq \sum_{i=1}^{N} I\left(s_{1, i}, s_{2, i}, \ldots, s_{K, i} ; y_{i}\right)
$$

where $R_{k, i}$ is defined as user $k$ 's mutual information on the $i^{\text {th }}$ subcarrier for a given input constellation. Therefore, it is equivalent to consider the problem of maximizing its upper bound $I_{\max }$, which is the sum of the joint mutual information at the right side. Furthermore, by using the mutual information chain rule, it can be decomposed as follows

$$
\begin{aligned}
I_{\max } & =\sum_{i=1}^{N} I\left(s_{1, i}, \ldots, s_{K, i} ; y_{i}\right) \\
= & \sum_{i=1}^{N} I\left(s_{\pi_{i}(1), i} ; y_{i}\right)+\ldots+ \\
& +\sum_{i=1}^{N} I\left(s_{\pi_{i}(K), i} ; y_{i} \mid s_{\pi_{i}(1), i}, \ldots, s_{\pi_{i}(K-1), i}\right)
\end{aligned}
$$

where vector $\Pi_{i}=\left[\pi_{i}(1), \ldots, \pi_{i}(K)\right]$ is a permutation of the integer vector $[1,2, \ldots, K]$ and represents the decoding order index on the $i^{\text {th }}$ subcarrier (users $\pi_{i}(1)$ and $\pi_{i}(K)$ being the first and last decoded users respectively). At the receiver side, the chain rule decomposition can be interpreted as applying successive interference cancellation (SIC) on every subcarrier, decoding from user $\pi_{i}(1)$ to $\pi_{i}(K)$. Indeed, assuming known the contribution of other users, the last decoded user is then able to remove multiuser interference and get a mutual information equal to the last sum of expression (2).

Let us add some notations to ease the introduction of power coefficients. The signal-to-interference-plus-noise ratio (SINR) of the first decoded user $\pi_{i}(1)$ can be expressed as follows

$$
\operatorname{SINR}_{\pi_{i}(1), i}=\frac{\left|h_{\pi_{i}(1), i}\right|^{2} p_{\pi_{i}(1), i}}{\sum_{j=2}^{K}\left|h_{\pi_{i}(j), i}\right|^{2} p_{\pi_{i}(j), i}+\sigma_{i}^{2}}
$$

Let $\mathbf{p}_{\mathbf{k}}=\left[p_{k, 1}, \ldots, p_{k, N}\right], \forall k=1 \ldots K$. We assume the number of users is sufficiently large. Then the sum of the joint mutual information expressed in (2) can be approximated as a function of all users' power allocation as below ${ }^{2}$

$$
\begin{aligned}
I_{\max } & =\mathcal{G}\left(\mathbf{p}_{1}, \ldots, \mathbf{p}_{K}\right) \\
& =\sum_{i=1}^{N} \Gamma_{\pi_{i}(1), i}\left(\operatorname{SINR}_{\pi_{i}(1), i}\right)+\sum_{i=1}^{N} \Lambda_{i}\left(\mathbf{p}_{-\pi_{i}(1), i}\right)
\end{aligned}
$$

where function $\Gamma_{\pi_{i}(1), i}($.$) denotes the mutual information of$ user $\pi_{i}(1)$ on the $i^{t h}$ subcarrier and $\Lambda_{i}($.$) denotes the joint$ mutual information of all remaining users (except user $\pi_{i}(1)$ ) on the $i^{t h}$ subcarrier, which is a function of these users' power

$$
\mathbf{p}_{-\pi_{i}(1), i}=\left[p_{\pi_{i}(2), i}, \ldots, p_{\pi_{i}(K), i}\right]
$$

\footnotetext{
${ }^{2}$ With this assumption, the interference seen by user $\pi_{i}(1)$ can be treated as Gaussian noise, therefore, his mutual information is a function of SINR.
}

It is obvious that function $\Lambda_{i}($.$) does not depend on p_{\pi(1), i}$, because the contribution of user $\pi_{i}(1)$ on the $i^{\text {th }}$ subcarrier is removed from $y_{i}$ at the first decoding step.

Following expression (3), different decoding orders can be used across channels. However, w.l.o.g. we assume a particular case where the first decoded user on all channels is the same user, the index of which is denoted $u$. This is expressed as

$$
\pi_{i}(1)=u, \quad i=1 \ldots N
$$

then (3) can be simplified to (4) as below, which will be useful for the proof in the next section.

$$
I_{\max }=\sum_{i=1}^{N} \Gamma_{u, i}\left(\operatorname{SINR}_{u, i}\right)+\sum_{i=1}^{N} \Lambda_{i}\left(\mathbf{p}_{-u, i}\right)
$$

Based on all presented above, the problem is therefore to find the optimal power allocation that maximizes the aggregate sum mutual information while satisfying constraint (1)

$$
\left[\mathbf{p}_{\mathbf{1}}^{\star}, \ldots, \mathbf{p}_{\mathbf{K}}^{\star}\right]=\arg \max _{\Sigma_{i=1}^{N} p_{k, i} \leq P, \forall k} \mathcal{G}\left(\mathbf{p}_{\mathbf{1}}, \ldots, \mathbf{p}_{\mathbf{K}}\right)
$$

It can be verified that the objective function is concave (this proof is not provided due to lack of space) and the related constraint is convex, which can be further simplified to

$$
\sum_{i=1}^{N} p_{k, i}=P \forall k
$$

The proof of this result will be provided in the next section.

\section{Multi-user Iterative Mercury/WATERfiLling ALGORITHM}

In this section, we assume perfect channel state information (CSI) at the receiver and transmitters. We propose an iterative power allocation algorithm which maximizes (3) following the steps of [5]. It is described as follows,

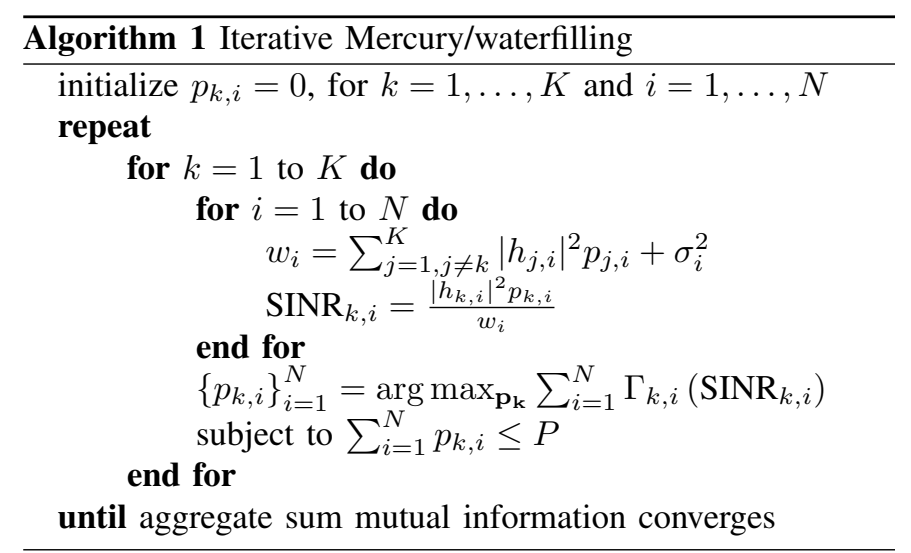

Each step of the multi-user iterative Mercury/waterfilling algorithm consists in finding the basic single-user Mercury/waterfilling solution for each user while regarding all other users signals as additional noise. In the following paragraphs, we prove the algorithm convergence and optimality. 


\section{A. Algorithm Convergence}

In this section, we will show that the multi-user iterative Mercury/waterfilling algorithm converges to the maximal aggregate sum mutual information. First, as shown in equation (4), when one does single-user Mercury/waterfilling for user $u$, the left part $\sum_{i=1}^{N} \Gamma_{u, i}\left(\operatorname{SINR}_{u, i}\right)$ is shown to be a non-decreasing function with respect to $\mathbf{p}_{\mathbf{u}}$, the right part $\sum_{i=1}^{N} \Lambda_{i}\left(\mathbf{p}_{-u, i}\right)$ is a constant with respect to $\mathbf{p}_{\mathbf{u}}$, and this holds true for any user $u=1, \ldots, K$. The multiuser objective is therefore non-decreasing with each singleuser Mercury/waterfilling step in the algorithm. Moreover, since each user has finite power constraint, there exists an upper-bound for the aggregate sum mutual information. This confirms the convergence.

\section{B. Convergence to the optimality}

We show in this section that by using multi-user iterative Mercury/waterfilling, the set $\left\{\mathbf{p}_{\mathbf{1}}, \ldots, \mathbf{p}_{\mathbf{K}}\right\}$ converges to the optimal values which maximize the aggregate sum mutual information. To prove the optimality part, we show that the necessary and sufficient condition of our optimization problem (5), is that each user applies single-user Mercury/waterfilling while taking multi-user interference as noise.

Theorem 1: In the context of $K$ users and $N$ parallel multiple access Gaussian channels, $\left\{\mathbf{p}_{\mathbf{1}}^{\star}, \ldots, \mathbf{p}_{\mathbf{K}}^{\star}\right\}$ is the optimal solution to the multi-user maximization problem

$$
\begin{array}{ll}
\operatorname{maximize} & \mathcal{G}\left(\mathbf{p}_{\mathbf{1}}, \ldots, \mathbf{p}_{\mathbf{K}}\right) \\
\text { subject to } & \sum_{i=1}^{N} p_{k, i} \leq P \forall k \\
& -p_{k, i} \leq 0 \forall k \forall i
\end{array}
$$

if and only if each user's power allocation is the single-user Mercury/waterfilling result while treating other users' signals as noise ${ }^{3}$.

1) Proof of necessary condition: Let us assume that at the global optimal point, only one user's (e.g. user $k$ ) power allocation would not satisfy the single-user Mercury/waterfilling condition: $\left\{\mathbf{p}_{1}^{\star}, \ldots, \mathbf{p}_{\mathbf{k}-\mathbf{1}}^{\star}, \mathbf{p}_{\mathbf{k}}, \mathbf{p}_{\mathbf{k}+\mathbf{1}}^{\star}, \ldots, \mathbf{p}_{\mathbf{K}}^{\star}\right\}$. In this case, according to (4), user $k$ 's power should satisfy

$$
\begin{aligned}
\mathbf{p}_{\mathbf{k}}^{\star} & =\arg \max _{\Sigma_{l=1}^{N} p_{k, l} \leq P} \mathcal{G}\left(\mathbf{p}_{\mathbf{1}}^{\star}, \ldots, \mathbf{p}_{\mathbf{k}-\mathbf{1}}^{\star}, \mathbf{p}_{\mathbf{k}}, \mathbf{p}_{\mathbf{k}+\mathbf{1}}^{\star}, \ldots \mathbf{p}_{\mathbf{K}}^{\star}\right) \\
& =\arg \max _{\sum_{l=1}^{N} p_{k, l} \leq P} \sum_{i=1}^{N} \Gamma_{k, i}\left(\mathrm{SINR}_{k, i}\right) \\
= & \arg \max _{\Sigma_{l=1}^{N} p_{k, l} \leq P} \sum_{i=1}^{N} \Gamma_{k, i}\left(\frac{\left|h_{k, i}\right|^{2} p_{k, i}}{\sum_{j=2}^{K}\left|h_{\pi_{i}(j), i}\right|^{2} p_{\pi_{i}(j), i}+\sigma_{i}^{2}}\right)
\end{aligned}
$$

The optimal result is obviously given by single-user Mercury/waterfilling algorithm, where user $k$ should consider other users' interference as noise, and therefore contradicts the initial assumption. This proof is true for any user $k=1, \ldots, K$. At

\footnotetext{
${ }^{3}$ with arbitrary input constellations, this last assumption is only valid when $K$ is large enough, which will ensure the overall interference from other $K-1$ users follows Gaussian distribution.
}

the global optimal point, each user should satisfy single-user Mercury/waterfilling condition, which is a necessary condition. In addition, at global optimal, single-user Mercury/waterfilling indicates that each user must allocate all its available power, providing therefore an explanation for power constraint (6).

2) Proof of sufficient condition: We will take advantage of the fact that Karush-Kuhn-Tucker (KKT) conditions [9] of the optimization problem is necessary and sufficient for optimality.

1. The Lagrangian function of the multi-user optimization problem is

$$
\begin{aligned}
& L\left(\left\{p_{k, i}\right\}_{k=1, i=1}^{K, N},\left\{\lambda_{k}\right\}_{k=1}^{K},\left\{\nu_{k, i}\right\}_{k=1, i=1}^{K, N}\right) \\
& \quad=\mathcal{G}\left(\mathbf{p}_{\mathbf{1}}, \ldots, \mathbf{p}_{\mathbf{K}}\right)-\sum_{k=1}^{K} \lambda_{k}\left(\sum_{i=1}^{N} p_{k, i}-P\right)+ \\
& \quad+\sum_{k=1}^{K} \sum_{i=1}^{N} \nu_{k, i} p_{k, i}
\end{aligned}
$$

and the multi-user KKT conditions are

$$
\begin{aligned}
\frac{\partial \mathcal{G}}{\partial p_{k, i}}-\lambda_{k}+\nu_{k, i} & =0, \quad \forall k \forall i \\
\lambda_{k} & \geq 0, \quad \forall k \\
\nu_{k, i} & \geq 0, \quad \forall k \forall i
\end{aligned}
$$

2. The Lagrangian function of the single-user (e.g. user $k$ ) optimization problem is

$$
\begin{aligned}
& L\left(\left\{p_{k, i}\right\}_{i=1}^{N}, \lambda_{k}^{\prime},\left\{\nu_{k, i}^{\prime}\right\}_{i=1}^{N}\right) \\
& =\sum_{i=1}^{N} \Gamma_{k, i}\left(\operatorname{SINR}_{k, i}\right)-\lambda_{k}^{\prime}\left(\sum_{i=1}^{N} p_{k, i}-P\right)+ \\
& +\sum_{i=1}^{N} \nu_{k, i}^{\prime} p_{k, i}
\end{aligned}
$$

and the single-user (user $k$ ) KKT conditions are

$$
\begin{aligned}
\frac{\partial \Gamma_{k, i}}{\partial p_{k, i}}-\lambda_{k}^{\prime}+\nu_{k, i}^{\prime} & =0, \forall i \\
\lambda_{k}^{\prime} & \geq 0, \\
\nu_{k, i}^{\prime} & \geq 0, \forall i
\end{aligned}
$$

It can be verified that the Slater's condition [9] is satisfied for the above two problems. Therefore, the strong duality holds, i.e. the KKT conditions provide necessary and sufficient conditions for optimality. From (3) we have

$$
\frac{\partial \mathcal{G}}{\partial p_{k, i}}=\frac{\partial \Gamma_{k, i}}{\partial p_{k, i}}
$$

and this equation holds for any user $k=1, \ldots, K$. Since multi-user KKT conditions (7) are equivalent to gathering each single-user KKT condition (8), global optimal is reached if and only if each user satisfies single-user Mercury/waterfilling treating other users' signals as noise. This confirms the optimality of iterative Mercury/waterfilling algorithm. 


\section{DECENTRALIZED ALGORITHM}

As we have shown above, multi-user iterative Mercury/waterfilling is the optimal power allocation scheme to maximize the sum of joint mutual information. However, when the number of user increases, implementation of algorithm 1 induces a large amount of overhead signaling to estimate all the channels. For this reason, we seek a decentralized power allocation scheme. In this section, we assume $K$ and $N$ are large enough, and $K / N \rightarrow \beta, 0<\beta \leq 1$.

Each step of algorithm 1 can be considered as a singleuser Mercury/waterfilling such that the current user (e.g. user $k$ ) tries to maximize his sum mutual information while treating other users' signals as background noise. So Mercury/waterfilling is performed based on user $k$ 's channel gain ${ }^{4}$ $\left\{\gamma_{k, 1}, \gamma_{k, 2}, \ldots, \gamma_{k, N}\right\}$,

$$
\gamma_{k, i}=\frac{\left|h_{k, i}\right|^{2}}{\sum_{j=1, j \neq k}^{K}\left|h_{j, i}\right|^{2} p_{j, i}+\sigma_{i}^{2}}
$$

which is a measure of the strength of his channels. Note that $\operatorname{SINR}_{k, i}=p_{k, i} \gamma_{k, i} \forall i$. The corresponding optimal result $\mathbf{p}_{k}^{*}=$ $\left[p_{k, 1}^{*}, \ldots, p_{k, N}^{*}\right]$ should satisfy [5]

$$
\begin{aligned}
p_{k, i}^{*} & =0 & & \gamma_{k, i} \leq \eta_{k} \\
\gamma_{k, i} \operatorname{MMSE}\left(p_{k, i}^{*} \gamma_{k, i}\right) & =\eta_{k} & & \gamma_{k, i}>\eta_{k}
\end{aligned}
$$

with $\eta_{k}$ such that the power constraint (6) is satisfied.

In the system model, when $K$ increases, the value of $\mathrm{SINR}_{k, i}$ is low due to the large interference enhancement from other $K-1$ users. Therefore, with the assumption of a large $K$, we can approximate the MMSE function by using the linear function in [5], [10]

$$
\begin{aligned}
\operatorname{MMSE}(\rho) & =1+\ddot{I}(0) \rho+\varepsilon\left(\rho^{2}\right) & & \rho \rightarrow 0 \\
& \approx 1+\ddot{I}(0) \rho & & \rho \rightarrow 0
\end{aligned}
$$

For simplicity let us assume $\eta_{k}=\eta \forall k$, and we use BPSK $(\ddot{I}(0)=-2)$ as input constellation. From (11), we obtain

$$
\gamma_{k, i}-2 \gamma_{k, i}^{2} p_{k, i}=\eta \quad \gamma_{k, i}>\eta
$$

Note that $\eta$ has a special meaning, it plays the role of a cut-off power threshold for channel gain $\gamma_{k, i}$. Let use define $\gamma_{c u t}=\eta$,

$$
\begin{array}{ll}
p_{k, i}>0 & \gamma_{k, i}>\gamma_{\text {cut }} \\
p_{k, i}=0 & \gamma_{k, i} \leq \gamma_{\text {cut }}
\end{array}
$$

From (10) and (13), we obtain

$$
p_{k, i}=\frac{\left(\gamma_{k, i}-\gamma_{c u t}\right)^{+}}{2 \gamma_{k, i}^{2}}
$$

which should satisfy the power constraint (6):

$$
\sum_{i=1}^{N} p_{k, i}=\sum_{i=1}^{N} \frac{\left(\gamma_{k, i}-\gamma_{c u t}\right)^{+}}{2 \gamma_{k, i}^{2}}=P
$$

\footnotetext{
${ }^{4}$ More precisely, it is based on user $k$ 's channel gain-to-noise-plusinterference ratio.
}

The interference seen by user $k$ on the $i^{\text {th }}$ subcarrier is

$$
\alpha_{k, i}=\sum_{j=1, \ell \neq k}^{K}\left|h_{j, i}\right|^{2} p_{j, i} \approx \sum_{j=1}^{K}\left|h_{j, i}\right|^{2} p_{j, i}
$$

which converges to the same constant $\alpha_{k, i}=\alpha \quad \forall k \forall i$ for a high number of users. Therefore, from (9) we obtain the channel gain approximation (assuming $\sigma^{2} \ll \alpha$ )

$$
\gamma_{k, i}=\frac{\left|h_{k, i}\right|^{2}}{\sigma^{2}+\alpha_{k, i}} \approx \frac{\left|h_{k, i}\right|^{2}}{\alpha}
$$

Put (17) into (14) and define: $\gamma_{c u t}=\frac{\left|h_{c u t}\right|^{2}}{\alpha}$, we obtain

$$
p_{k, i}=\alpha \frac{\left(\left|h_{k, i}\right|^{2}-\left|h_{c u t}\right|^{2}\right)^{+}}{2\left|h_{k, i}\right|^{4}}
$$

and put (18) into (15), we have

$$
\alpha=\frac{P}{\sum_{i=1}^{N} \frac{\left(\left|h_{k, i}\right|^{2}-\left|h_{c u t}\right|^{2}\right)^{+}}{2\left|h_{k, i}\right|^{4}}}
$$

also put (18) into (16),

$$
\begin{gathered}
\alpha=\sum_{k=1}^{K}\left|h_{k, i}\right|^{2} \alpha \frac{\left(\left|h_{k, i}\right|^{2}-\left|h_{c u t}\right|^{2}\right)^{+}}{2\left|h_{k, i}\right|^{4}} \\
\sum_{k=1}^{K} \frac{\left(\left|h_{k, i}\right|^{2}-\left|h_{c u t}\right|^{2}\right)^{+}}{\left|h_{k, i}\right|^{2}}=2
\end{gathered}
$$

and let $M$ be the number of active (powered-on) subcarriers among $K$ subcarriers, we derive

$$
\begin{gathered}
\sum_{k^{\prime}=1}^{M} \frac{\left(\left|h_{k^{\prime}, i}\right|^{2}-\left|h_{c u t}\right|^{2}\right)^{+}}{\left|h_{k^{\prime}, i}\right|^{2}}=2 \\
\left|h_{c u t}\right|^{2}=\frac{M-2}{\sum_{k^{\prime}=1}^{M} \frac{1}{\left|h_{k^{\prime}, i}\right|^{2}}}
\end{gathered}
$$

Based on (13)-(20), the following algorithm is obtained.

Algorithm 2 Multi-user Mercury/waterfilling using MMSE approximation

1. For arbitrary $i$, sort $\left\{h_{1, i}, \ldots, h_{K, i}\right\}$ in decreasing order to get $\left\{h_{1, i}^{\prime}, \ldots, h_{K, i}^{\prime}\right\}$.

2. Initialize $m=2$.

3. For the same $i$, Do $m=m+1$, find $m$ until it satisfies: $\left|h_{m, i}^{\prime}\right|^{2} \geq \frac{m-2}{\sum_{k=1}^{m} 1 / \mid h_{k, i}^{\prime}} \geq\left|h_{m+1, i}^{\prime}\right|^{2}$.

3. Use (20) to calculate $\left|h_{\text {cut }}\right|^{2}$, let $M=m$.

4. Use (19) to calculate multi-user interference $\alpha$.

5. Use (18) to calculate $\left\{p_{k, i}\right\}_{i=1}^{N}$.

Furthermore, in order to handle a fully decentralized algorithm, each user $k$ should be able to assign his power $P$ without the knowledge of other users' channel coefficients. However, as we can see, steps 1 and 4 require each user $k$ to have this knowledge on the $i^{t h}$ subcarrier. These two steps thus cannot be done by user $k$ himself without any feedback. One solution to avoid this feedback and to make 
the algorithm completely decentralized is the following: in the large number of user $K$ and subcarriers $N$ case (assuming all users' channel coefficients follow the same distribution related to the environment), equation (20) can be rewritten as

$$
\left|h_{c u t}\right|^{2}=\frac{M-2}{\sum_{k^{\prime}=1}^{M} \frac{1}{\left|h_{k, i}^{\prime}\right|^{2}}} \approx \frac{M / \beta-2}{\sum_{i=1}^{M / \beta} \frac{1}{\left|h_{k, i}^{*}\right|^{2}}}
$$

where $\left|h_{k, i}^{*}\right|^{2}$ is the $i^{\text {th }}$ element of user $k$ 's coefficients $\left\{\left|h_{k, 1}\right|^{2}, \ldots,\left|h_{k, N}\right|^{2}\right\}$ sorted in the decreasing order.

\section{NUMERICAL RESULTS}

In this section, numerical results are presented to validate our theoretical claims. We consider parallel multiple access Gaussian channels with $N(=500)$ subcarriers and $K(=\beta N)$ users while QPSK is taken as unique input constellation. For all simulations, the system load $\beta$ varies from 0.1 to 1 . The SNR is defined as $P / \sigma^{2}$, where $P(=1)$ is the single user power constraint and $\sigma^{2}$ is the Gaussian noise of variance 0.1 . The SNR is set to $10 \mathrm{~dB}$.

In Fig. 2, we show the convergence behavior of iterative Mercury/waterfilling algorithm. The spectral efficiency (bit/subcarrier) versus the system load $\beta$ are plotted for five iterations. As expected, one can easily observe the convergence.

In Fig. 3, we show the comparison of spectral efficiencies obtained with iterative Mercury/waterfilling (algorithm 1 ), uniform power allocation (each user allocates the same power $P / N$ on all subcarriers) and the proposed decentralized scheme (algorithm 2). With regard to channel knowledge, the related upper bound and lower bound are provided by iterative Mercury/waterfilling and uniform power allocation respectively, since the former needs perfect channel knowledge and the latter does not need any channel knowledge at all. Thus, we define the increase of maximum sum mutual information rate as power allocation gain, this increase being due to applying a smarter power allocation strategy. As expected, in Fig. 3, both strategies (algorithms 1 and 2) offer up to $80 \%$ power allocation gain, while the decentralized scheme (algorithm 2) reaches more than $90 \%$ of the upper bound.

\section{CONCLUSION}

In this paper, Mercury/waterfilling policy has been extended to a multi-user context. We found the optimal power allocation that achieves the maximum sum mutual information of parallel multiple access Gaussian channels under the assumption of arbitrary fixed input constellations and individual power constraints. We showed that the multi-user objective is a non-decreasing function with respect to the power allocation of single-user Mercury/waterfilling performed while treating other users' signals as noise. We proved the convergence and optimality of iterative Mercury/waterfilling algorithm. A decentralized power allocation algorithm was proposed based on the MMSE approximation. We found that the spectral efficiency of this algorithm had a satisfying result with respect to that of the uniform power allocation and close to the optimal, which was shown in the simulation results.

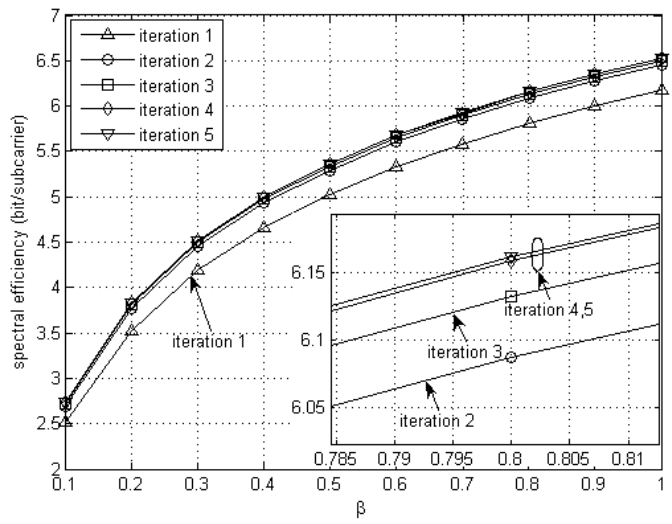

Fig. 2. Convergence of iterative Mercury/waterfilling.

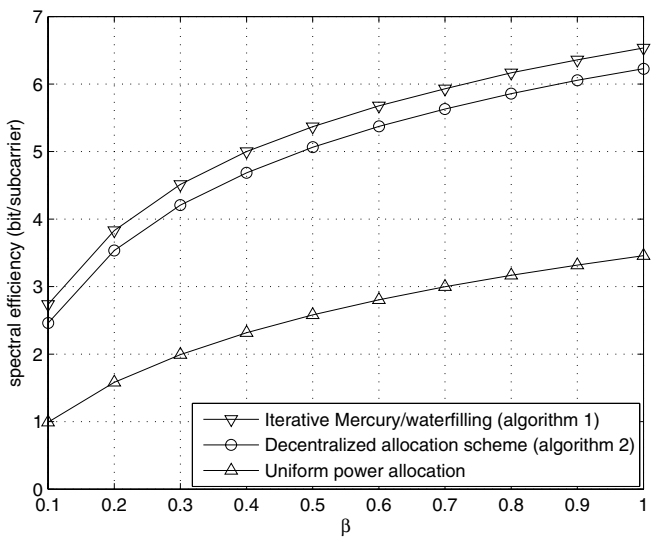

Fig. 3. Spectral efficiency comparison.

\section{REFERENCES}

[1] J. Jang and K. B. Lee, "Transmit Power Adaptation for Multiuser OFDM Systems", IEEE Journal on Selected Areas in Communications, vol. 21, no. 2, pp. 171-178, Feb. 2003.

[2] C. Y. Wong, R. S. Cheng, K. B. Letaief and R. D. Murch, "Multiuser OFDM with adaptive sub-carrier, bit, and power allocation", IEEE Journal on Selected Areas in Communications, vol. 17, no. 10, pp. 1747-1758, Oct. 1999.

[3] K. Kim, Y. Han and S. L. Kim, "Joint subcarrier and power allocation in uplink OFDMA systems", IEEE Communication Letters, vol. 9, no. 6, pp. 526-528, Jun. 2005.

[4] T. M. Cover and J. A. Thomas, Elements of Information Theory, New York: Wiley, 1991.

[5] A. Lozano, A. M. Tulino and S. Verdú, "Optimum Power Allocation for Parallel Gaussian Channels with Arbitrary Input Distributions," IEEE Trans. on Information Theory, vol. 52, no. 7, pp. 3033-3015, Jul. 2006.

[6] W. Yu, W. Rhee, S. Boyd and J. M. Cioffi, "Iterative Water-filling for Gaussian Vector Multiple Access Channels," IEEE Trans. on Information Theory, vol. 50, pp. 145-152, Jan. 2004.

[7] R. Ahlswede, "Multi-way Communication Channels," in 2nd Inter. Symp. Information Theory, pp. 103-105, 1971.

[8] H. Liao, "Multiple Access Channels," Ph.D thesis, University of Hawaii, 1972.

[9] S. Boyd and L. Vandenberghe, Convex Optimization, Cambridge University Press, 2004.

[10] D. Guo, S. Shamai and S. Verdú, "Mutual Information and Minimum Mean-Square Error in Gaussian Channels," IEEE Trans. on Information Theory, vol. 51, pp. 1261-1282, Apr. 2005. 